Pathologe 2008 · [Suppl 2] 29:374-378

DOI 10.1007/s00292-008-1075-2

Online publiziert: 16. Oktober 2008

(c) Springer Medizin Verlag 2008

\author{
S. Lax ${ }^{1} \cdot$ A. Lebeau ${ }^{2} \cdot$ A. Schneider ${ }^{3}$ \\ ${ }^{1}$ Institut für Pathologie, LKH Graz West, Graz, Österreich \\ ${ }^{2}$ Institut für Pathologie, Universitätsklinikum Hamburg-Eppendorf \\ ${ }^{3}$ Klinik für Gynäkologie, Charité-Universitätsmedizin Berlin, \\ Campus Benjamin Franklin und Campus Mitte
}

\section{Sitzungsbericht der AG Gynäko- und Mammapathologie}

\author{
Anlässlich der 92. Jahrestagung \\ der Deutschen Gesellschaft \\ für Pathologie in Berlin
}

\section{Allgemeine Einleitung}

\section{S. Lax, Graz}

Die Arbeitsgemeinschaft für Gynäkound Mammapathologie möchte bewusst einen Bogen „from bench to bedside and microscope" spannen und versucht daher, eine Synthese von molekularer Forschung und klinisch orientierter Diagnostik zu unterstützen. Deshalb wird seit mehreren Jahren das Programm ihrer Sitzung im Rahmen der Jahrestagung der DGP in Form einer Mischung aus aktueller Wissenschaft und wesentlichen Aspekten für die Praxis ausgerichtet. Dies wird einerseits durch freie Vorträge sowie andererseits durch Übersichtsreferate zu aktuellen Themen, gehalten von eingeladenen Referenten, erzielt. Dadurch konnte die Zuhörerzahl bei den Sitzungen wesentlich gesteigert und auch vermehrt Kolleg(inn)en aus dem niedergelassenen Bereich angesprochen werden. In diesem Jahr beschäftigten sich die freien Vorträge mit Forschungsthemen aus dem Bereich Mamma (8 Vorträge), Ovar (2 Vorträge), Endometrium und Vulva (je 1 Vortrag). Sie zeigten zum Teil einen starken Praxisbezug (z. B. methodische Untersuchungen für dieHer2/neu-Bestimmungbzw. Mikro- kalke beim Mammascreening) und behandelten andererseits auch neue Aspekte aus der molekularen Forschung (beispielsweise Amplifikation des ÖstrogenrezeptorGens beim Endometriumkarzinom).

Das Schwerpunktthema beschäftigte sich mit der Bedeutung der intraoperativen Diagnostik in der Gynäko- und Mammapathologie. Unter dem Motto „Pathologie im Zentrum des operativen Geschehens" wurde diese Thematik in Form von 4 Referaten sowohl aus Sicht der Klinik bzw. der Pathologie abgehandelt. Dankenswerterweise übernahm Herr Prof. Dr. Achim Schneider (Universitätsfrauenklinik der Charité Campus Benjamin Franklin und Campus Mitte) beide klinischen Vorträge. Diese sind nachfolgend ebenso wie die von Frau PD Dr. Annette Lebeau und mir gehaltenen Referate kurz zusammengefasst.

Kein anderer Bereich der diagnostischen Pathologie bringt den Pathologen in eine solche Nähe zum Patienten wie die intraoperative Diagnostik (Schnellschnitt). Die diagnostischen Aussagen haben unmittelbare Konsequenz und beeinflussen das weitere operative Geschehen ganz entscheidend. Der Schnellschnittdiagnostik sind technische und methodische Grenzen auferlegt, dennoch er- fordert sie von den Pathologen Entscheidungsfreudigkeit und großes Verantwortungsbewusstsein. Somit ist die intraoperative Gewebediagnostik eine klinische Tätigkeit. Um eine hohe Qualität in der Schnellschnittdiagnostik zu erreichen, muss dieser ein entsprechender Stellenwert im Rahmen des Faches Pathologie eingeräumt werden. Im Rahmen der Schnellschnittdiagnostik bilden wir Pathologen mit unseren operativ tätigen Kollegen ein Team, in dem beide Seiten in ihrer Tätigkeit aufeinander angewiesen sind und das daher möglichst gut funktionieren muss. Um Operateuren und Pathologen eine intensive Kommunikation zu ermöglichen und die Transportzeiten zu minimieren, sollte der Schnellschnittbereich idealerweise in unmittelbarer Nähe zum Operationsbereich gelegen sein. Dadurch werden optimale Voraussetzungen für eine erfolgreiche Diagnostik und Therapie geschaffen. 


\section{Bedeutung des Schnellschnittes bei Brusttumoren aus Sicht der Operateure}

\section{A. Schneider, Berlin}

Gerade bei der Behandlung von Frauen mit Brustkrebs ist die enge Kooperation zwischen Klinikern und Pathologen unabdingbar: Radiologen, Pathologen und Operateure arbeiten ab dem Zeitpunkt der Verdachtsdiagnose eng zusammen und sprechen kontinuierlich miteinander.

Mehr als 90\% aller Tumoren sind primär durch Stanzbiopsie gesichert, wobei der Schnellschnitt an der Stanze nicht empfohlen wird. Die Dignität der Läsion ist damit bereits präoperativ abgeklärt und bekannt. Auch Größe und Ausdehnung eines Tumors sollten bereits vor der Operation durch die Stanze evaluiert werden und nur in Ausnahmefällen intraoperativ durch den Schnellschnitt zu beurteilen sein. Die Sicherheitsabstände zu den Resektionsrändern werden intraoperativ durch Radiographie und Sonographie des Operationspräparates und Vergleich mit der präoperativen Bildgebung evaluiert. Nur in Ausnahmefällen werden die Resektionsränder durch den Pathologen im Schnellschnitt beurteilt. Voraussetzung dafür ist, dass die Läsion im Präparat palpabel und im Durchmesser größer als $10 \mathrm{~mm}$ ist.

Die Domäne der Schnellschnittdiagnostik bei Brustkrebs ist die intraoperative Untersuchung der Sentinel-Lymphknoten, da nur im positiven Fall die einzeitige Operation der Axilla erfolgt. Hierbei muss man wissen, dass die Zuverlässigkeit des Schnellschnitts bei 90\% liegt, wobei die Sensitivität für die Detektion von Makrometastasen $98 \%$ beträgt [1]

Wird beim Nachweis von Mikrometastasen oder isolierten Tumorzellen im Paraffinschnitt und bei vorher negativem Sentinel-Lymphknoten im Schnellschnitt die axilläre Lymphonodektomie komplettiert, so sind bei $96 \%$ der Frauen die restlichen Lymphknoten frei von Makrometastasen [1].

Die Beurteilung des Sentinel-Lymphknotens im Schnellschnitt ist aufwendig und vor allem bei invasivem lobulärem Karzinom in mehr als 20\% der Fälle

Tab. 1 Diagnostische Genauigkeit der intraoperativen Schnellschnittuntersuchung beim Mammakarzinom

\begin{tabular}{|c|c|c|c|c|}
\hline Indikation & $\begin{array}{l}\text { Treffsicherheit } \\
\text { (\%) }\end{array}$ & Sensitivität ${ }^{\mathrm{b}}(\%)$ & Spezifität` (\%) & Literatur \\
\hline Dignität & $98-99$ & $92-99$ & $93-100$ & {$[12,19,20]$} \\
\hline \multicolumn{5}{|l|}{ Resektionsrandstatus: } \\
\hline Nur Makroskopie & 70 & 82-94 & $61-88$ & {$[6,12]$} \\
\hline Mit Gefrierschnitt & 84-100 & $59-91$ & $86-100$ & $\begin{array}{l}{[7,8,10,17,18,} \\
21]\end{array}$ \\
\hline $\begin{array}{l}\text { Sentinel-Lymph- } \\
\text { knoten-Befall }\end{array}$ & $84-91$ & $\begin{array}{l}\text { Makrometas- } \\
\text { tasen: } 92-100 \\
\text { Mikrometastasen: } \\
17-52\end{array}$ & $98-100$ & {$[9,11,13,15,22]$} \\
\hline \multicolumn{5}{|c|}{$\begin{array}{l}{ }^{a} \text { Treffsicherheit: richtig positiv + richtig negativ/Gesamtzahl } \\
\text { bSensitivität: richtig positiv/(richtig positiv + falsch negativ) } \\
\text { 'Spezifität: richtig negativ/(richtig negativ + falsch positiv). }\end{array}$} \\
\hline
\end{tabular}

falsch negativ. Daher wird der molekularbiologische Nachweis von Tumorzellen in den nächsten Jahren den konventionellen Schnellschnitt verdrängen. Mammaglobin und der Nachweis von Zytokeratin-mRNA zeigen eine hohe Sensitivität für die Detektion von Metastasen mit einem Durchmesser von mehr als $2 \mathrm{~mm}[2,3]$. Die intraoperative Untersuchung von Lymphknoten auf CK19-mRNA ergab in keinem Fall ein falsch positives Ergebnis [4].

Neben der Beratung durch den Radiologen und Operateur möchten mehr und mehr Patientinnen mit Brustkrebs auf die Expertise des Pathologen zurückgreifen. Hier wird der direkte Dialog von der Patientin gesucht. Was erwartet sie vom $\mathrm{Pa}-$ thologen? Neben der bildlichen Erklärung der Biologie ihres Tumors benötigt sie menschliche Zuwendung. Pathologen sind für diesen Dialog noch nicht optimal vorbereitet und müssen hier für die $\mathrm{Zu}$ kunft gerüstet sein. Dies stärkt einerseits die Rolle der Pathologie, erhöht andererseits das Wissen der Patientin und ermöglicht ihr mehr Sicherheit und Vertrauen in ihre Behandlung.

\section{Schnellschnitt bei Mammatumoren aus der Sicht der Pathologie}

\section{A. Lebeau, Hamburg}

Die Indikationen zur intraoperativen Schnellschnittuntersuchung bei Mammatumoren haben sich im Laufe der letzten Jahre gewandelt. Dies ist die Folge der Etablierung präoperativer minimal in- vasiver Diagnostikverfahren und veränderter operativer Therapiestrategien. Die intraoperative Dignitätsbeurteilung mithilfe des Schnellschnittes soll heute nur noch in Ausnahmefällen erfolgen [5, 14]. Derzeit wird die Schnellschnittuntersuchung hierzulande in erster Linie als Option für die intraoperative Beurteilung der Resektionsränder (RR) am Mammaexzidat und/oder die Beurteilung der Sentinel-Lymphknoten (SLN) genutzt. Wird intraoperativ ein Karzinombefall der RR oder SLN nachgewiesen, ermöglicht dies zumindest in einem Teil der Fälle ein einzeitiges operatives Vorgehen.

Grundsätzlich stellt sich zur Abschätzung von Nutzen und Risiken der intraoperativen Schnellschnittuntersuchung die Frage nach der diagnostischen Genauigkeit. Einen Überblick bezogen auf die verschiedenen Indikationen gibt (• Tab. 1). Bei der Beurteilung der Dignität liegt die Treffsicherheit sehr hoch. Dennoch besteht die Gefahr der Fehlinterpretation seltener oder schwieriger Läsionen (z. B. sklerosierende Läsion, Adenom der Mamille, malignes Lymphom). Eine hohe Prozessqualität dient der Vermeidung möglicher Fehlerquellen wie „sampling error" oder mangelhafte Schnittqualität.

Die diagnostische Genauigkeit der intraoperativen RR-Beurteilung hängt von der Methode ab. Die geringste Spezifität besitzt die rein makroskopische Beurteilung. Demgegenüber lässt sich durch eine Gefrierschnittuntersuchung eine deutlich höhere Spezifität erreichen. Die Daten zur Sensitivität zeigen eine hohe Schwankungsbreite. Die Gründe hierfür könnten 
zum einen in der Heterogenität der Art der Gewebeentnahme für den Schnellschnitt liegen (Entnahme senkrecht zum RR am Exzidat versus tangential versus Schnellschnitt an zusätzlichem Biopsat aus der Exzisionshöhle), zum anderen in den Unterschieden hinsichtlich der geforderten Sicherheitsabstände in den verschieden Studien.

Die Schnellschnittuntersuchung der Sentinel-Lymphknoten besitzt eine hohe Treffgenauigkeit bei der Entdeckung von Makrometastasen (>2 mm). Demgegenüber wird bei einmaligem Anschnitt der halbierten oder lamellierten SLN im Schnellschnitt nur die Minderheit der Mikrometastasen (>0,2 und $\leq 2 \mathrm{~mm}$ ) entdeckt.

Der Einsatz der Telepathologie zur intraoperativen Schnellschnittbeurteilung erfordert nach derzeitigem Stand die Gewährleistung des Facharztstandards vor Ort.

\section{Fazit}

Die intraoperative Schnellschnittuntersuchung stellt eine sensitive und spezifische Methode zur Dignitätsbestimmung bei palpablen Tumoren der Mamma dar. Die intraoperative Beurteilung der Resektionsränder ermöglicht die einzeitige Exzision mit ausreichenden Sicherheitsabständen bei 60-90\% der Mammakarzinome. Die Schnellschnittuntersuchung des Sentinel-Lymphknotens führt zur intraoperativen Erkennung von mindestens 92\% der Makrometastasen und eröffnet damit die Möglichkeit der einzeitigen Lymphknotendissektion. Die Sensitivität hinsichtlich der Entdeckung von Mikrometastasen ist deutlich geringer und begründet im Wesentlichen die notwendigen Zweiteingriffe.

\section{Bedeutung des Schnellschnittes bei gynäkologischen Tumoren aus der Sicht der Operateure}

\section{A. Schneider, Berlin}

Um zu wissen, was sie von den Pathologen erwarten können, müssen die Kliniker verstehen, wo die Grenzen der Pathologie liegen. Grundvoraussetzung dafür ist, dass sich alle Kliniker Kenntnisse in der Pathologie erwerben und diese kon- tinuierlich im Dialog mit den Pathologen erneuern.

\section{Gebärmutterhals}

Der Schnellschnitt spielt bei der Diagnostik und Therapie der Präkanzerosen der Cervix uteri keine wichtige Rolle. Nur in Ausnahmefällen wird man ein Zervixbiopsat oder eine Konusspitze vom Pathologen im Schnellschnitt beurteilen lassen [23]. Der Kliniker weiß, dass die Aussagekraft über den Schweregrad der Läsion und die Resektionsgrenzen am im Paraffin eingebetteten Gewebe viel größer ist.

Bei Gebärmutterhalskrebs wird die Ausdehnung der Erkrankung chirurgisch gestaged. Am wichtigsten ist der Lymphknotenstatus, der laparoskopisch erhoben werden kann. Ist der Tumor im Durchmesser kleiner als $2 \mathrm{~cm}$, ist das SentinelKonzept sinnvoll und mit einer Sensitivität von 90\% und einem negativen Vorhersagewert von 99\% auch zuverlässig [24].

Die Rate an falsch negativen Schnellschnittergebnissen bei Sentinel-Lymphknoten liegt bei 3,5\% [25].

Für den Nachweis von zirkulierenden Tumorzellen oder die molekularbiologische intraoperative Untersuchung von Sentinel-Lymphknoten ist beim Zervixkarzinom der Marker CK19-mRNA nicht geeignet, sondern hier stellt HPV-RNA den sensitivsten und spezifischsten Marker dar [26].

Frauen mit Kinderwunsch und einem unter $2 \mathrm{~cm}$ großen Tumor kann ein Erhalt des Uterus und der Fertilität durch radikale Trachelektomie ermöglicht werden. Der Resektionsstatus kann bei Längsschnitt durch das Trachelektomiepräparat nur dann im Schnellschnitt beurteilt werden, wenn makroskopisch eine Läsion sichtbar ist [27].

Sind bei Frauen mit fortgeschrittenem Tumor pelvine und/oder paraaortale Lymphknoten im Schnellschnitt tumorbefallen, wird die Lymphonodektomie komplettiert. Der Uterus verbleibt aber in situ und es erfolgt die primäre Radio-Chemo-Therapie. Im Schnellschnitt sollte der oberste paraaortale Lymphknoten beurteilt werden: Ist er befallen, erfolgt automatisch die Entfernung der SkalenusLymphknoten links.

Zeigen sich im Rahmen der StagingLaparoskopie verdächtige intraperitoneale
Läsionen, so werden diese biopsiert und dem Schnellschnitt zugeführt. Bei nachgewiesener intraperitonealen Aussaat ist die Fortsetzung der Operation wenig sinnvoll, sondern die primäre Chemotherapie, ggf. kombiniert mit Hyperthermie, wird eingeleitet.

Beim Rezidiv und geplanter Exenteration nach Gebärmutterhalskrebs wird ebenfalls ein paraaortaler Lymphknotenbefall oder eine intraperitoneale Aussaat durch Schnellschnitt ausgeschlossen. Zudem wird bei der Exenteration idealerweise das Präparat dem Pathologen direkt übergeben, der durch Schnellschnitt den Resektionsstatus evaluieren kann. Bei nachgewiesener R1-Resektion kann dann das betroffene Areal nachreseziert und intraoperativ bestrahlt werden, oder es können Clips angebracht werden, die postoperativ eine gezielte Boostbestrahlung erlauben. Durch die laparoskopischhistopathologische Evaluierung kann bei der Hälfte der Exenterationskandidatinnen durch den intraoperativen Schnellschnitt die unnötige Laparotomie vermieden werden [28].

\section{Gebärmutterkörper}

Invasionstiefe, Grading, Befall der Zervix und Lymphknotenstatus sind prä- und intraoperativ die wichtigsten Prognoseparameter. Mehrere Studien zeigen, dass die intraoperative Beurteilung von Invasionstiefe und Grading unzuverlässig ist $[29,30]$.

Hinsichtlich des Gradings und der Invasionstiefe korreliert der Gefrierschnitt mit der endgültigen Histologie nur in ca. $60 \%$ der Fälle, bis zu 40\% werden in der endgültigen histologischen Untersuchung im Grading höher eingestuft. Auch lässt das Ergebnis des Gefrierschnittes bezüglich Infiltrationstiefe und Grading keine sichere Aussage darüber zu, welche Patientin ein erhöhtes Risiko für Lymphknotenmetastasen hat und daher lymphonodektomiert werden sollte [31]. Eine KostenNutzen-Analyse zeigt, dass auch für Frauen mit hochdifferenziertem Endometriumkarzinom (Grading 1) das komplette chirurgische Staging einschließlich Lymphonodektomie am effektivsten ist [32]. 


\section{Eierstock}

Die wichtigsten Fragen an den Pathologen beim Schnellschnitt sind: benigne versus Borderline versus invasiv sowie Primärtumor versus Metastase.

Beim intraoperativen Schnellschnitt liegt für Borderline-Tumoren die Rate an Überdiagnosen bei 7\% und Unterdiagnosen bei 30\% [33]. Die Unterscheidung Borderline versus benigne Ovarialtumoren gelingt mit hoher Zuverlässigkeit, Gleiches gilt für die Unterscheidung benigne versus maligne Ovarialtumoren. Hier liegt die Domäne des Schnellschnitts [33, 34].

Zur Unterscheidung zwischen primärem Ovarialtumor und Tumormetastase kann der Schnellschnitt nur in ca. 80\% der Fälle eine korrekte Diagnose liefern [35].

Die Differenzierung des Tumortyps, z. B. Karzinom versus Keimzell- versus Keimstrangtumor, stellt üblicherweise kein intraoperatives Problem dar, da es für die chirurgische Ausdehnung der Operation keine Konsequenz hat, welche der drei Tumorarten vorliegt. Die Behandlung ist mit einer Entfernung des inneren Genitales, der Lymphknoten, des großen Netzes und der Entnahme multipler Peritonealbiopsien identisch. Daher ist der Schnellschnitt für diese Fragestellung nicht indiziert.

\section{Vulva}

Invasionstiefe und Lymphknotenbefall, in diesem Fall speziell der Befall der Sentinel-Lymphknoten, sind die beiden wichtigsten Fragen an den Pathologen. Ob die Invasionstiefe $1 \mathrm{~mm}$ oder mehr beträgt, sollte am Paraffinschnitt entschieden werden. Dieses Ergebnis entscheidet über die Durchführung oder Nichtdurchführung der Lymphonodektomie. Für die Evaluierung des Status der inguinalen Lymphknoten ist das Sentinel-Konzept inzwischen bei Frauen mit Vulvakarzinom klinisch etabliert [36]. Wichtig ist, dass bei tumorbefallenem Sentinel-Lymphknoten die komplette Lymphonodektomie ipsilateral und kontralateral erfolgen muss.

\section{Fazit}

Nur der kontinuierliche Dialog zwischen Klinikern und Pathologen prä-, intra- und postoperativ sichert eine hohe Therapiequalität bei der Behandlung von Frauen mit gynäkologischen Tumoren. Dieser
Dialog darf nicht nur auf Befundberichten beruhen, sondern muss direkt - persönlich und am Mikroskop - erfolgen.

\section{Der Schnellschnitt bei Uterus- und Ovarialtumoren aus der Sicht der Pathologie}

\section{S. Lax, Graz}

Aus Sicht der Pathologie ist ein Schnellschnitt dann indiziert, wenn dessen Ergebnis eine unmittelbare therapeutische Konsequenz hat. Dadurch können vor allem weitere operative Eingriffe vermieden werden. Dies setzt aber die volle Aufklärung der Patienten und alle erforderlichen technischen, medizinischen und logistischen Möglichkeiten voraus, um eine Operation auch in vollem Umfang durchführen zu können. Ziel jeder Schnellschnittuntersuchung ist daher eine möglichst hohe Trefferquote, vor allem hinsichtlich der therapieentscheidenden Einordnung einer Läsion. Dabei steht die Festlegung der Dignität über einer exakten Typisierung bzw. dem Grading. In Zentren sollte jeder Tumor intraoperativ sofort nach Absetzen des Organs an die Pathologie übersandt werden, um eine Asservierung von Frischgewebe für die Tumorbank und eine optimale Fixierung des Operationspräparates (für eine hohe Qualität der Paraffinhistologie) zu ermöglichen.

Bei Tumoren des Uterus (Endometrium bzw. Zervix) sollte eine möglichst exakte Tumordiagnose bereits vor der Operation in Form einer Paraffinhistologie vorliegen, da sie das weitere therapeutische Vorgehen ganz wesentlich bestimmt. Eine Schnellschnittdiagnostik von Abradaten, Biopsaten und Konus ist hingegen nur in Ausnahmefällen zulässig, nämlich bei dringender Notwendigkeit einer einzeitigen Operation. Dem Schnellschnitt bleibt bei Tumoren des Endometriums die Feststellung der Eindringtiefe in das Myometrium, die Bestimmung einer Zervixinfiltration bzw. einer extrauterinen Aussaat vorbehalten. Beim Endometrium- wie beim Zervixkarzinom kommt der Untersuchung der pelvinen Lymphknoten im Schnellschnitt dann eine Bedeutung zu, wenn davon die Durchführung einer paraaortalen Lymphaden- ektomie abhängt. Die Untersuchung der Lymphknoten ist sehr aufwendig, nicht zuletzt aufgrund der zeitintensiven makroskopischen Bearbeitung. Meist werden mehrere Lymphknotenstationen untersucht, wobei vor allem der Lymphknotengruppe an der Arteria und Vena iliaca communis eine besondere Bedeutung zukommt. Die Sensitivität der Lymphknoten im Schnellschnitt liegt bei ca. $80 \%$, die Spezifität und der positive Vorhersagewert bei $100 \%$, der negative Vorhersagewert bei 90\% [37]. Ähnliche Ergebnisse zeigten sich auch nach neoadjuvanter Radio-ChemoTherapie des Zervixkarzinoms [38].

Beim Endometriumkarzinom stimmt die Beurteilung der Invasionstiefe in 6795\% der Fälle überein, wobei zum Teil auch die makroskopische Beurteilung durch den Operateur in der Literatur vorgeschlagen wird. Diese Rolle kommt unserer Meinung nach aber nicht zuletzt aus Gründen der Qualitätssicherung dem Pathologen zu. Die Beurteilung des Differenzierungsgrades im Schnellschnitt zeigt in $58 \%$ der Fälle eine Übereinstimmung mit der definitiven Paraffinhistologie [39].

Bei Ovarialtumoren bestehen abgesehen von der Laparoskopie keine Möglichkeiten einer präoperativen Biopsie, weiters nur eine eingeschränkte Aussagekraft bildgebender Verfahren. Daher ist der Schnellschnitt die einzige Methodik, um ein einzeitiges operatives Vorgehen zu gewährleisten. Bei den Ovarialtumoren haben die Operateure daher eine besonders hohe Erwartung an die Treffsicherheit des Schnellschnittes. Die höchste Trefferquote weisen maligne Ovarialtumoren mit bis zu 100\% auf, die geringste Borderline-Tumoren mit ca. 60\% [40]. Die Problematik bei den Borderline-Tumoren liegt vor allem in der Treffsicherheit bei der Auswahl der Proben, da aus zeitlichen Gründen und Gründen der Verfügbarkeit von ausreichendem Material für die Paraffinhistologie nur wenige Proben im Schnellschnitt untersucht werden sollten (als Faustregel maximal drei Proben). Schwierigkeiten kann auch die Diagnostik seltener Tumoren, wie z. B. Keimzelltumoren, maligne Lymphome, und die Abgrenzung von Metastasen gegenüber primären Ovarialkarzinomen bereiten [41]. Die Genauigkeit der Schnellschnitte im Ovar liegt bei etwa 60-97\% [42]. Dabei wurden 
auch falsch positive Fälle (bis zu 11\%) genannt. Bei bis zu 6\% der Ovarialtumoren muss die Entscheidung auf die Paraffinhistologie verschoben werden [42]. Auch die Diagnose einer kleinherdigen Invasion bei Borderline-Tumoren kann schwierig sein. Als wesentliche Unterstützung im diagnostischen Entscheidungsprozess werden eine intraoperative Zweitmeinung sowie die Steigerung der Erfahrung durch gemeinsame Diskussion problematischer Fälle erachtet (kontinuierlicher Verbesserungsprozess). Für das Erkennen von $\mathrm{Me}$ tastasen können die Bilateralität sowie die Größe hilfreich sein [43].

\section{Korrespondenzadresse \\ Prof. Dr. S. Lax}

Institut für Pathologie, LKH Graz West

Göstingerstraße 22, 8020 Graz, Österreich

sigurd.lax@meduni-graz.at

Interessenkonflikt. Der korrespondierende Autor gibt an, dass kein Interessenkonflikt besteht.

\section{Literatur}

1. Langer I, Guller U, Berclaz G et al. (2008) Accuracy of frozen section of sentinel lymph nodes: a prospective analysis of 659 breast cancer patients of the Swiss multicenter study. Breast Cancer Res Treat (Epub ahead of print)

2. Valeria D, Daniela A, Fernando P et al. (2008) Sentinel lymph node molecular pathology in breast carcinoma. Diagn Mol Pathol (Epub ahead of print)

3. Viale G, Dell'Orto P, Biasi MO et al. (2008) Comparative evaluation of an extensive histopathologic examination and a real-time reverse-transcriptionpolymerase chain reaction assay for mammaglobin and cytokeratin 19 on axillary sentinel lymph nodes of breast carcinoma patients. Ann Surg 247: 136-142

4. Tsujimoto M, Nakabayashi K, Yoshidome K et al. (2007) One-step nucleic acid amplification for intraoperative detection of lymph node metastasis in breast cancer patients. Clin Cancer Res 13: 4807-4816

5. Albert U-S (2008) (Hrsg) Stufe-3-Leitlinie Brustkrebsfrüherkennung in Deutschland. Zuckschwerdt, München

6. Balch GC, Mithani SK, Simpson JF, Kelley MC (2005) Accuracy of intraoperative gross examination of surgical margin status in women undergoing partial mastectomy for breast malignancy. Am Surg 71: 22-27

7. Cabioglu N, Hunt KK, Sahin AA et al. (2007) Role for intraoperative margin assessment in patients undergoing breast-conserving surgery. Ann Surg Oncol 14: 1458-1471

8. Cendan JC, Coco D, Copeland EM III (2005) Accuracy of intraoperative frozen-section analysis of breast cancer lumpectomy-bed margins. J Am Coll Surg 201: 194-198

9. Chao C, Wong SL, Ackermann D et al. (2001) Utility of intraoperative frozen section analysis of sentinel lymph nodes in breast cancer. Am J Surg 182: 609615
10. Cox CE, Ku NN, Reintgen DS et al. (1991) Touch preparation cytology of breast lumpectomy margins with histologic correlation. Arch Surg 126: 490 493

11. Creager AJ, Geisinger KR, Shiver SA et al. (2002) Intraoperative evaluation of sentinel lymph nodes for metastatic breast carcinoma by imprint cytology. Mod Pathol 15: 1140-1147

12. Fleming FJ, Hill AD, Mc Dermott EW et al. (2004) Intraoperative margin assessment and re-excision rate in breast conserving surgery. Eur J Surg Oncol 30: 233-237

13. Grabau DA, Rank F, Friis E (2005) Intraoperative frozen section examination of axillary sentinel lymph nodes in breast cancer. APMIS 113: 7-12

14. Kreienberg R, Kopp I, Albert US et al. (2008) Interdisziplinäre S3-Leitlinie für die Diagnostik, Therapie und Nachsorge des Mammakarzinoms. Zuckschwerdt, München

15. Langer I, Guller U, Berclaz G et al. (2008) Accuracy of frozen section of sentinel lymph nodes: a prospective analysis of 659 breast cancer patients of the Swiss multicenter study. Breast Cancer Res Treat (Epub ahead of print)

16. Niu Y, Fu XL, Yu Y et al. (2007) Intra-operative frozen section diagnosis of breast lesions: a retrospective analysis of 13,243 Chinese patients. Chin Med J (Engl) 120: 630-635

17. Noguchi M, Minami M, Earashi M et al. (1995) Pathologic assessment of surgical margins on frozen and permanent sections in breast conserving surgery. Breast Cancer 2: 27-33

18. Olson TP, Harter J, Munoz A et al. (2007) Frozen section analysis for intraoperative margin assessment during breast-conserving surgery results in low rates of re-excision and local recurrence. Ann Surg Oncol 14: 2953-2960

19. Scheiden R, Sand J, Tanous AM et al. (2001) Accuracy of frozen section diagnoses of breast lesions after introduction of a national programme in mammographic screening. Histopathology 39: 74-84

20. Sultana N, Kayani N (2005) Validity of frozen section in the diagnosis of breast lumps: 5 years experience at the Aga Khan University Hospital. J Pak Med Assoc 55: 533-536

21. Weber S, Storm FK, Stitt J, Mahvi DM (1997) The role of frozen section analysis of margins during breast conservation surgery. Cancer J Sci Am 3: 273277

22. Weiser MR, Montgomery LL, Susnik B et al. (2000) Is routine intraoperative frozen-section examination of sentinel lymph nodes in breast cancer worthwhile? Ann Surg Oncol 7: 651-655

23. Behtash N, Karimi Zarchi M, Hamedi B et al. (2007) The value of frozen sectioning for the evaluation of resection margins in cases of conization. Arch Gynecol Obstet 276: 529-532

24. Altgassen C, Hertel H, Brandstadt A et al. (2008) Multicenter validation study of the sentinel lymph node concept in cervical cancer: AGO Study Group. J Clin Oncol 26: 2943-2951

25. Hertel H, Köhler C, Grund D et al. (2006) Radical vaginal trachelectomy (RVT) combined with laparoscopic pelvic lymphadenectomy: prospective multicenter study of 100 patients with early cervical cancer. Gynecol Oncol 103: 506-511

26. Häfner N, Gajda M, Altgassen C et al. (2007) HPV16-E6 mRNA is superior to cytokeratin 19 mRNA as a molecular marker for the detection of disseminated tumour cells in sentinel lymph nodes of patients with cervical cancer by quantitative reverse-transcription PCR. Int J Cancer 120: 1842-1846
27. Tanguay C, Plante M, Renaud MC et al. (2004) Vaginal radical trachelectomy in the treatment of cervical cancer: the role of frozen section. Int J Gynecol Pathol 23: 170-175

28. Köhler C, Tozzi R, Possover M et al. (2002) Explorative laparoscopy prior to exenterative surgery. Gynecol Oncol 86: 311-315

29. Case AS, Rocconi RP, Straughn JM Jr et al. (2006) A prospective blinded evaluation of the accuracy of frozen section for the surgical management of endometrial cancer. Obstet Gynecol 108: 1375-1379

30. Sanjuan A, Cobo T, Pahisa J et al. (2006) Preoperative and intraoperative assessment of myometrial invasion and histologic grade in endometrial cancer: role of magnetic resonance imaging and frozen section. Int J Gynecol Cancer 16: 385-390

31. Frumovitz M, Slomovitz BM, Singh DK et al. (2004) Frozen section analyses as predictors of lymphatic spread in patients with early-stage uterine cancer. J Am Coll Surg 199: 388-393

32. Cohn DE, Huh WK, Fowler JM et al. (2007) Cost-effectiveness analysis of strategies for the surgical management of grade 1 endometrial adenocarcinoma. Obstet Gynecol 109: 1388-1395

33. Tempfer CB, Polterauer S, Bentz EK et al. (2007) Accuracy of intraoperative frozen section analysis in borderline tumors of the ovary: a retrospective analysis of 96 cases and review of the literature. Gynecol Oncol 107: 248-252

34. Medeiros LR, Rosa DD, Edelweiss Ml et al. (2005) Accuracy of frozen-section analysis in the diagnosis of ovarian tumors: a systematic quantitative review. Int J Gynecol Cancer 15: 192-202

35. Stewart CJ, Brennan BA, Hammond IG et al. (2005) Accuracy of frozen section in distinguishing primary ovarian neoplasia from tumors metastatic to the ovary. Int J Gynecol Pathol 24: 356-362

36. Van der Zee AG, Oonk MH, De Hullu JA et al. (2008) Sentinel node dissection is safe in the treatment of early-stage vulvar cancer. J Clin Oncol 26: 884-889

37. Scholz HS, Lax SF, Benedicic C et al. (2003) Accuracy of frozen section examination of pelvic lymph nodes in patients with FIGO stage IB1 to IIB cervical cancer. Gynecol Oncol 90: 605-609

38. Bader AA, Winter R, Moinfar F et al. (2006) Is intraoperative frozen section analysis of pelvic lymph nodes accurate after neoadjuvant chemotherapy in patients with cervical cancer? Gynecol Oncol 103: 106-112

39. Case AS, Rocconi RP, Straughn JM Jr et al. (2006) A prospective blinded evaluation of the accuracy of frozen section for the surgical management of endometrial cancer. Obstet Gynecol 108: 1375-1379

40. Twaalfhoven FC, Peters AA, Trimbos JB et al. (1991) The accuracy of frozen section diagnosis of ovarian tumors. Gynecol Oncol 41: 189-192

41. Medeiros LR, Rosa DD, Edelweiss MI et al. (2005) Accuracy of frozen-section analysis in the diagnosis of ovarian tumors: a systematic quantitative review. Int J Gynecol Cancer 15: 192-202

42. Acs $G$ (2002) Intraoperative consultation in gynecologic pathology. Semin Diagn Pathol 19: 237254

43. Seidman JD, Kurman RJ, Ronnett BM (2003) Primary and metastatic mucinous adenocarcinomas in the ovaries: incidence in routine practice with a new approach to improve intraoperative diagnosis. Am J Surg Pathol 27: 985-993 